\title{
Philosophiques
}

\section{Raymond Montpetit : Comment parler de la littérature? \\ Montréal, HMH, Les Cahiers du Québec, collection \\ "Philosophie ", 1976, 195 p.}

\section{Claude Panaccio}

Volume 5, numéro 1, avril 1978

URI : https://id.erudit.org/iderudit/203089ar

DOI : https://doi.org/10.7202/203089ar

Aller au sommaire du numéro

Éditeur(s)

Société de philosophie du Québec

ISSN

0316-2923 (imprimé)

1492-1391 (numérique)

Découvrir la revue

Citer cet article

Panaccio, C. (1978). Raymond Montpetit : Comment parler de la littérature?

Montréal, HMH, Les Cahiers du Québec, collection « Philosophie ", 1976, 195 p.

Philosophiques, 5(1), 155-171. https://doi.org/10.7202/203089ar d'utilisation que vous pouvez consulter en ligne.

https://apropos.erudit.org/fr/usagers/politique-dutilisation/ 


\section{ETUDES CRITIQUES}

Raymond MONTPETIT : Comment parler de la littérature? Montréal, HMH, Les Cahiers du Québec, collection "Philosophie ", 1976, 195 p.

\section{par Claude Panaccio}

D'être soulevée, la question "Comment parler de la littérature?" suscite aussitôt chez le lecteur une certaine frayeur et un certain vertige. Il songe à la multitude des débats qui depuis plusieurs années déjà hantent les pages de revues spécialisées comme Poétique, Tel Quel, Communications, Langages, ou de ceux (les mêmes le plus souvent) que reprennent inlassablement, de façon toujours plus sophistiquée et plus complexe, les colloques ou les congrès consacrés à la sémiologie de l'œuvre littéraire ou à l'analyse textuelle. Il pense à l'herméneutique, à la grammatologie, à la sémanalyse, à la poétique, à l'analyse structurale du récit, à la sémantique structurale, à la stylistique, à la phonostylistique, à la morphosyntaxe, à la sociologie de la littérature, et, pourquoi pas ? à la critique des idéologies. Il imagine des bibliographies monstrueuses et des labyrinthes conceptuels réservés à de petites sectes d'initiés.

Or le livre de Raymond Montpetit est loin d'être abstrus. L'" honnête homme ", amateur de littérature, s'y retrouvera sans trop de difficulté. En un sens, on pourrait y voir un ouvrage de vulgarisation, mais ce serait en restreindre injustement la portée. Car si l'on $y$ trouve des présentations simples et accessibles de Schleiermacher, de Dilthey, de Ricœur, de Barthes, de Todorov, $\mathrm{du}$ formalisme russe et de certaines tendances récentes en critique littéraire, l'intérêt de l'entreprise réside dans la façon synthétique et heuristique dont tout cela est mis en relation, rattaché à un nombre restreint de grandes orientations, dont l'opposition caractérise non seulement le discours méta-littéraire, mais peut-être de 
façon beaucoup plus générale le discours théorique contemporain dans son entier.

C'est ainsi que Montpetit distingue trois façons principales de "parler de la littérature ": la manière "herméneutique ", la manière "formaliste " et la manière "machiniste ", tripartition qui fournit à l'ouvrage son plan général. On pourrait à gros traits résumer l'intrigue du livre de la façon suivante : jusqu'ici - du moins au cours de ce siècle - le discours sur la littérature a été partagé entre les deux premières tendances qui constituent, selon l'expression de Michel Foucault, "les deux grandes formes d'analyse de notre âge $"^{1}$. Mais voici qu'apparaît depuis quelque temps une troisième approche, encore peu théorisée, mais apparemment plus susceptible de saisir la spécificité du texte littéraire, celle que l'auteur lui-même privilégie et qu'il appelle l'approche " machiniste".

Je voudrais ici d'abord présenter brièvement la façon dont Montpetit caractérise les trois approches en question, puis scruter de plus près la question de la spécificité du machinisme, en particulier dans son rapport au formalisme. Je suggérai à ce propos que la façon dont l'auteur traite cette question prête quelque peu à confusion en rabattant les uns sur les autres un certain nombre de problèmes théoriquement distincts.

\section{Interpréter, formaliser, machiner}

La critique littéraire n'est pas, sur le plan théorique, une entreprise innocente. Les questions qu'elle adresse à l'œuvre, la façon dont elle en localise et en repère le sens, l'ensemble des catégories qu'elle utilise, tout cela déjà est grevé de philosophie. Et c'est ce que Montpetit montre particulièrement bien pour le cas de l'approche "interprétative " ou " herméneutique ", celle qui vise à refaire en sens inverse le chemin parcouru par l'expression créatrice et à remonter du produit fini (l'œuvre) à l'élan intentionnel qui l'a engendré, à retrouver donc la subjectivité derrière les mots.

Pour dévoiler les particularités théoriques de ce genre d'approche, Montpetit nous conduit d'abord vers des philosophes: Schleiermacher, Dilthey, et surtout Paul Ricœur. Il montre ensuite que certaines pratiques de critique littéraire peuvent être directe-

1. FOUCAULT, M., Les Mots et les choses, Paris, Gallimard, 1966, p. 312 (cité par Montpetit, p. 9). 
ment rattachées à ce genre de philosophie. Le cas type est celui de Georges Poulet, mais l'auteur nous entretient aussi au passage de Doubrovsky, de Starobinski, de Jean Rousset, de Jean-Pierre Richard et de quelques autres.

La critique herméneutique est ainsi caractérisée par sept traits (p. 42-48), que je ne reprendrai pas ici, mais qui au bout du compte dépendent tous de ceci que l'œuvre y est abordée comme expression d'une subjectivité. Le critique intervenant lui aussi dans le processus à titre de sujet, littérature et critique littéraire deviennent un cas particulier de l'intersubjectivité, ce qui, selon Montpetit, comporte l'inconvénient majeur de laisser échapper la spécificité du texte comme objet non subjectif.

L'approche formaliste prend à peu près exactement le contrepied de la précédente : les sept traits par lesquels elle sera à son tour qualifiée (p. 109-110) s'opposent un à un aux sept caractéristiques de l'herméneutique. L'idée centrale ici est que le traitement formaliste met la subjectivité entre parenthèses pour s'attaquer à la structuration interne du signifiant. Là où il était question de communication, d'expression, de compréhension, figurent maintenant des codes, des systèmes, des lois, des règles de distribution. En même temps qu'il échappe à l'intersubjectivité, le littéraire "s'horizontalise ". Au bout du compte, les formalistes visent la construction d'une science de la littérarité, capable d'en expliciter les lois générales de constitution et d'organisation.

Les principaux auteurs considérés sont: d'abord Roland Barthes, - la partie qui lui est consacrée est d'ailleurs remarquable et constitue l'un des sommets de l'ouvrage, - ensuite les formalistes russes et finalement Tzvetan Todorov, le projet d'une science de la littérarité s'exprimant dans ces deux derniers cas de façon très explicite.

L'auteur, on le sent, est beaucoup plus sympathique à cette seconde voie qu'à la première, mais, au bout du chapitre, il se révèle de nouveau insatisfait, et presque pour la même raison : si l'approche formaliste permet - du moins en principe - de qualifier le littéraire dans sa différence d'avec les autres pratiques signifiantes, elle perd pourtant la spécificité de chaque texte, de chaque œuvre, en les aplatissant tous dans la " littérarité ". Son vouloir de scientificité, nous dit Montpetit, est "réducteur des différences" 
(p. 118). D'où la question qui, on s'en aperçoit maintenant, guidait depuis le début toute l'entreprise de l'auteur :

"Peut-il y avoir un traitement du texte dont le but serait de mettre à jour le travail particulier qui a justement donné lieu à ce texte-ci et non point à un autre?" (p. 119, souligné par l'auteur).

La troisième partie du livre, celle qui porte sur le "machinisme", propose à cette question une réponse affirmative.

Mais une surprise nous attend ici : six pages seulement, réparties en trois groupes de deux (p. 123-125, 150-152 et 169 171 ), sont consacrées à expliciter de manière générale en quoi consiste l'approche machiniste, le reste du chapitre étant occupé par deux exemples : une analyse du Quatuor d'Alexandrie de Lawrence Durrell (p. 125-150) et une analyse d'Otbello de Shakespeare (p. 152-169). Pour une raison qu'il n'explique pas, l'auteur a choisi de montrer le machinisme à l'œuvre plutôt que de le théoriser. Mais puisqu'il faut au moins minimalement le caractériser, disons, avec Montpetit, que la gestion machiniste ne cherche ni le sujet créateur derrière l'œuvre, ni les lois générales qui la régissent de haut, mais qu'elle entend dégager les rouages internes du fonctionnement d'un texte particulier.

Dans le cas du Quatuor, ce fonctionnement est conçu sur le modèle du travail du rêve, tel que Freud l'a décrit, c'est-à-dire essentiellement comme jeu de substitutions : "transformation, déformation, transcription, traduction, condensation, déplacement, figuration, distorsion (...). Tous ces mots nous conduisent à l'idée d'une substitution" (p. 124). L'analyse machinique doit ainsi repérer, à différents niveaux, la présence dans l'œuvre de séries substitutives, le passage d'un terme à l'autre dans la série conditionnant en quelque sorte la poursuite du récit : les narrateurs se substituent les uns aux autres, les versions les unes aux autres, les partenaires amoureux les uns aux autres et ainsi de suite ; la trame du roman n'est rien d'autre que l'entrelacement de ces divers déplacements.

Bien qu'il n'y soit pas explicitement fait référence, sauf dans la bibliographie, on trouverait sans doute la formulation la plus élaborée de ce modèle dans Logique du sens de Gilles Deleuze²,

2. DELeUZE, G., Logique du sens, Paris, Éd. de Minuit, 1969. 
ouvrage qui cherche précisément, mais de façon toute informelle, à mettre en place une logique des séries substitutives qui constituerait la logique générale du sens. Deleuze et Montpetit se rencontrent en particulier en ceci qu'ils posent tous deux l'impossibilité de principe de clore les séries substitutives. La machination littéraire, écrit Montpetit, "comme celle du rêve dont elle se réclame, en est une de substitutions, substitutions dont on ne parvient jamais à toucher l'origine » (p. 125). Phénomène étrange, qui est particulièrement bien mis en lumière à propos du Quatuor d'Alexandrie, où finalement la "vraie " version des faits demeure inaccessible.

On reconnaît là au passage la problématique " derridaienne " de la " trace $"^{3}$, celle de la "différance " (avec un " a ") ${ }^{4}$, celle du " déplacement ". C'est-à-dire, pour être plus clair, qu'on retrouve ici la thèse caractéristique de ce que j'appellerais la philosophie post-structuraliste française : les signifiants, en particulier ceux de l'écriture, se renvoient indéfiniment les uns aux autres, sans qu'il soit jamais possible de totaliser le sens $s^{6}$. L'absence de théorisation du machinisme dans le livre de Montpetit reconduit ainsi le lecteur - par un phénomène d'intertextualité typique - d'une part à Freud (L'interprétation des rêves), d'autre part à Deleuze, Derrida, Barthes, Kristeva et compagnie.

\section{Du rapport entre le formel et le machinique}

L'écart entre le traitement des deux premières parties et celui de la troisième paraît de prime abord significatif. Il faut admettre que si le "machinisme" est privilégié, son statut théorique demeure fort obscur. Alors notamment que les deux premières approches sont rigoureusement mises en parallèle et par là en opposition l'une avec l'autre, leur rapport avec la troisième reste énigmatique, réduit à une métaphore laconique : l'herméneutique fouille sous le texte et le formalisme le surplombe, tandis que le machinisme se tient dans le texte'.

\footnotetext{
3. Cf. Derrida, J., De la grammatologie, Paris, Éd. de Minuit, 1967, surtout p. 65-108.

4. Cf. Derrida, J., "La différence ", in Marges de la philosophie, Paris, Éd. de Minuit, 1972, p. $1-29$.

5. Cf. le titre fort significatif de l'ouvrage de Stephen HeAtH, consacré à Barthes, Vertige du déplacement, (Paris, Fayard, 1974).

6. $\dot{A}$ mon sens, c'est précisément cette thèse qui empêche à l'heure actuelle le "poststructuralisme" de convoler avec le marxisme, et cela malgré un flirt évident.

7. Cf. en particulier p. 151-152. Cette tripartition n'est pas sans rappeler la distinction proposée par Deleuze dans Logique du sens (p. 152-158) entre trois sortes de philosophes : les philosophes de la hauteur (les platoniciens), ceux de la profondeur (les présocratiques) et ceux de la surface (les stö̈ciens). Inutile de dire que Deleuze est lui-même partisan du troisième groupe.
} 
Il y aurait déjà à propos du rapport entre machinisme et herméneutique quelques questions à soulever. Plusieurs formules de Montpetit suggèrent l'existence d'une téléologie de la machine ${ }^{8}$, et le mot même de "machine ", qui d'après Larousse se définit comme "Appareil combiné pour produire certains effets", semble bien renforcer une telle suggestion. L'auteur pourtant nous assure que la machine textuelle n'est nullement soumise au sujet créateur. Mais alors qui donc l'a si savamment construite? D'où lui vient sa finalité ? Questions naïves sans doute, mais qu'il n'est pas si facile d'évacuer.

Laissons-les de côté cependant pour nous arrêter plus longuement sur la question - à mon sens plus intéressante - du rapport entre le machinisme et le formalisme. Pour Montpetit, l'opposition se résume à ceci : le formalisme cherche les lois universelles de la littérarité, le machinisme entend décrire les rouages de tel texte particulier. Deux corollaires importants accompagnent cette façon d'opérer la distinction :

1) le formalisme s'exprime dans un métalangage, alors que le machinisme laisse jouer le discours de l'œuvre ${ }^{10}$;

2) le formalisme occulte le travail du lecteur alors que le machinisme, reconnaissant avec Barthes le " pluriel du texte ", admet que : "on peut toujours faire du texte un autre usage "; un autre usage, c'est-à-dire une autre lecture.

8. Cf. par exemple les passages suivants : " Le texte peut donc s'analyser rouage par rouage, et se révéler alors être une machine savamment montée pour produire ses effets ..." (p. 12) ; "le texte, une machine qui est montée pour produire certains effets..." (p. 151); "ce que le traitement machinique dégage, ce sont plutôt les mécanismes montés de faģon à assurer la marche du texte, la production de cette écriture et des effets qu'elle veut provoquer " (p. 170). (Souligné par moi).

9. Cf. par exemple : "le texte est ici décrit par un discours d'un autre type que le sien, discours qui croit possible de parler à distance, et de loin, comme d'un autre lieu qui, fort probablement, serait le lieu surélevé du 'méta' ". (p. 105) ; "La différence des langages, c'est la possibilité du métalangage, la possibilité d'un discours formaliste sur la littérature " (p. 106); "Le traitement formaliste (...) n'est possible qu'en tant qu'un discours $X$ peut maîtriser le jeu d'un discours Y qu'il n'est pas" (p. 107).

10. Cf. par exemple : "Le machiniste entretient avec son texte un rapport opérationnel qui le place de plain-pied avec son texte" (p. 151) ; "Ces discours sur la littérature (I'herméneutique et le formalisme) se déterminent comme métalangage (...). Le machiniste est par rapport à cette topologie, hors lieu; son travail opère le texte et s'effectue au niveau même de cette écriture particulière dans laquelle il est pris". (p. 152). 
Je dois dire que pour ma part je ne trouve pas cette opposition lumineuse. D'abord le formalisme y est presque caricaturé : perçu comme cette entreprise qui cherche à " dominer "le discours littéraire, à le "surplomber ", à le "maîtriser ", il se voit soupçonné d'une inquiétante volonté de puissance. L'idée même d'un métalangage devient ainsi antipathique, et le privilège accordé au machinisme tient pour une large part à cet effet rhétorique : il est plus sympathique, n'est-ce pas? de se tenir au niveau de... et de jouer avec..., que de contraindre ou de soumettre à des lois.

Pareille manœuvre d'ailleurs s'esquissait dès le chapitre II, consacré précisément à l'examen du formalisme. La moitié du chapitre en effet - la première moitié qui plus est - porte exclusivement sur Roland Barthes. Or Montpetit montre lui-même, et je partage cette interprétation, que Barthes n'est pas un formaliste:

"partie d'une position herméneutique, la démarche barthésienne n'a fait qu'effleurer ou qu'esquisser une méthodologie formaliste, celle-ci éclatant au moment où elle aurait pu se constituer, sous la pression du pluriel" (p. 93).

Le formalisme se trouve ainsi escamoté avant même que d'être entré en scène : le passage sur Barthes se termine sur cette conclusion (étrange) : il faut détruire le métalangage (p. 97).

Bien sûr, la seconde partie du chapitre nous entretient aussi du formalisme russe et de la poétique de Todorov, mais l'insistance, par ailleurs justifiée, à caractériser ces entreprises par la recherche d'une légalité universelle du littéraire, oblige à faire l'économie d'une analyse plus précise de la démarche formelle et des tentatives dans lesquelles elle se manifeste avec le plus de clarté, celle par exemple de l'analyse automatique de discours.

Mais je voudrais surtout suggérer que la façon dont Montpetit oppose formalisme et machinisme tend à produire une confusion entre au moins trois problèmes distincts, alors que le véritable problème que soulèvent des analyses comme celles que Montpetit poursuit lui-même à propos de Durrell et de Shakespeare n'est ni l'un ni l'autre de ces trois-là, mais un quatrième qui n'est presque pas touché par l'auteur. 


\section{De quatre problèmes qu'il ne faut pas confondre}

Les quatre problèmes auxquels je songe sont les suivants :

1) le problème du métalangage,

2) le problème de la légalité (c'est-à-dire de l'universel et du particulier),

3) le problème du rôle du lecteur,

4) le problème du rapport formel entre fonctionnement textuel et structure linguistique.

Je les reprendrai ici un à un.

\subsection{Faut-il détruire les métalangages?}

Répondre à cette question par l'affirmative, ce serait récuser du même coup cette autre question qui donnait son titre à l'ouvrage : "Comment parler de la littérature ?"; ce serait poser tout simplement qu'il ne faut pas parler de la littérature. Pour qu'un discours soit identifié comme métadiscours, ne suffit-il pas en effet qu'il parle d'un autre discours? On sait, par exemple, que pour Jakobson même une explication fournie par un locuteur sur le sens de ce qu'il vient de dire constitue une manifestation de la fonction métalinguistique $^{11}$. De ce point de vue, il n'est nullement requis que le métalangage soit en tous points distinct du langage-objet : l'un et l'autre peuvent par exemple recourir à une langue commune. Mais il suffit que l'on soit capable au moyen du métalangage de renvoyer à certains éléments au moins du langageobjet. Il n'y a donc là rien de très mystérieux, ni de très menaçant, mais simplement le nom très général d'un ensemble de pratiques discursives, au demeurant fort diversifiées, dont certaines sont d'usage quotidien.

Pour peu qu'on souscrive à un objectif minimal de rigueur, on admettra sans doute qu'il y a quelque intérêt à définir de façon un peu précise les catégories dans lesquelles on parlera de la littéra-

11. Cf. JaKoBson, R., Essais de linguistique générale (t.I), Paris, Éd. de Minuit, 1963, p. 217. 218. ". . le métalangage n'est pas seulement un outil scientifique nécessaire à l'usage des logiciens et des linguistes; il joue aussi un rôle important dans le langage de tous les jours (...). Chaque fois que le destinateur et/ou le destinataire jugent nécessaire de vérifier s'ils utilisent bien le même code, le discours est centré sur le code, il remplit une fonction métalinguistique. " 
ture ou de l'œuvre littéraire, et à spécifier les règles de leur utilisation. Cette opération justement n'est rien d'autre que la construction d'un métalangage! Montpetit, dans son "Ouverture ", déclare espérer que son livre contribuera " à la constitution de discours sur la littérature qui voudront maîtriser leurs propres fondements et rendre leurs analyses rigoureuses" (p. 5-6). Je ne sais pas trop ce que c'est pour un discours que de "maîtriser ses propres fondements ", mais je crois tout de même voir poindre ici le projet d'un métalangage.

Si d'ailleurs nous relisons l'analyse ("machiniste") que Montpetit consacre au Quatuor d'Alexandrie, nous y verrons à l'œuvre toute une série de catégories proprement métalinguistiques. J'en mentionne - dans un ordre arbitraire - quelquesunes parmi celles, d'ailleurs beaucoup plus nombreuses, qui figurent entre les pages 125 et 134 :

$\begin{array}{llll}\text { - titre } & - \text { adverbe } & - \text { écriture } & \text { - épigraphe } \\ \text { - exergue } & - \text { temps des verbes } & - \text { déplacement } & - \text { procédé } \\ \text { - citation } & - \text { énonciation } & - \text { métaphore } & - \text { transitivité } \\ \text { - substitution } & \text { - narrateur } & - \text { hiérarchie } & - \text { instance } \\ \text { - texte } & - \text { récit } & - \text { intertextualité } & \text { etc... }\end{array}$

Un métalangage, si je ne me trompe, n'est rien d'autre qu'un tel ensemble de catégories (lexique), accompagné des règles de leur combinaison et de leur application (syntaxe et sémantique). Le fait que ces règles demeurent tacites dans le texte de Montpetit, c'est-àdire que ni la syntaxe ni la sémantique du métalangage machiniste ne soient rendues explicites, n'enlève rien au fait que de telles règles sont agissantes, et dans ce texte même.

Il y a quelque chose de mythologique et de mystificateur à parler de la destruction du métalangage. J'y crois reconnaître à vrai dire comme un relent de cette vieille volonté métaphysique d'entrer dans les choses mêmes. Et bien sûr, c'est là une illusion : le machiniste n'échappera pas plus que l'herméneute ni que le formaliste à la nécessité du métalangage. La véritable opposition entre la deuxième et la troisième partie du livre de Montpetit ne se situe pas ici, ou alors elle est illusoire.

\subsection{L'universel contre le particulier}

Si la distinction formalisme/machinisme ne tient pas à la présence ou à l'absence d'un métalangage, peut-être dépend-elle du 
type de métalangage employé et des objectifs qui lui sont assignés. Or c'est bien sur une opposition de ce genre que Montpetit insiste le plus volontiers : le formaliste s'intéresse à la "littérarité ", c'està-dire à des universaux, le machiniste à des textes littéraires, c'està-dire à des individus.

L'auteur cite à ce propos certains passages très explicites de Todorov, considéré ici comme représentant typique du formalisme, par exemple celui-ci :

"L'objet de la science n'est pas, n'a jamais été un objet réel, pris tel qu'en lui-même. Ce ne sont donc pas, dans le cas des études littéraires, les œuvres littéraires elles-mêmes ${ }^{12}$.

"Il en résulte, commente Montpetit, qu'il n'y a pas de poétique particulière à une ouvre littéraire, mais seulement une poétique de la littérarité » (p. 115) ; laquelle voudrait énoncer des lois générales, alors que le machiniste, lui, vise à décrire les "rouages" de telle ou telle ouvre littéraire particulière. Le formalisme est du côté de la science, le machinisme s'apparente davantage à la critique littéraire ${ }^{13}$. Les limites du formalisme sont donc celles de toute science, puisque, comme chacun sait, "il ne peut y avoir de science que du général" (p. 116). Le machiniste, au contraire, en abandonnant les prétentions scientifiques d'un Todorov, peut se permettre de transgresser allégrement ces limites et de rejoindre la singularité du texte-objet.

L'évidence de cette distinction, et d'ailleurs de l'adage séculaire qui la soutient, est.cependant sujette à caution. Il y aurait par exemple quelque chose d'étrange à dire qu'un biologiste ne peut pas décrire un état particulier d'un organisme particulier parce que la biologie est une science, ou que, pour la même raison, la météorologie ne peut pas - en principe - prédire le temps qu'il fera demain. Il est certain que les catégories scientifiques sont abstraites, mais leur intérêt est précisément de pouvoir servir à l'analyse de situations concrètes. Qu'il y ait toujours nécessairement un résidu, cela ne doit pas nous traumatiser outre mesure, puisque c'est là le sort de toute entreprise discursive, qu'elle soit scientifique

12. TODOROV, T., "Roman Jakobson poéticien ", Poétique, no 7, 1971, p. 228 (cité par Montpetit, p. 115).

13. Cf. la distinction opérée par Barthes entre sience de la littérature et critique littéraire, in Critique et vérité, Paris, Éd. du Seuil, 1966, p. 56-75. 
ou non. On ne peut pas raisonnablement espérer relayer le discours scientifique par un autre qui serait plus approprié à la description de l'individuel.

Considérons à ce propos quelques passages des analyses « concrètes " proposées par Montpetit dans la dernière partie du livre:

«Alors que aoriste, imparfait et plus-que-parfait sont, comme le montre Benveniste, des temps de l'énonciation historique (. . .), présent, futur et parfait établissent une liaison entre le dit d'une part, et d'autre part le moment et la personne qui dit " (p. 128 129, souligné par l'auteur).

"Un roman de tel genre se doit de produire nécessairement tels et tels effets" (p. 151).

". . . un impératif qui est catégorique pour tout personnage (. . .), celui de la poursuite du récit (. . ), le personnage doit agir pour que le récit progresse" (p. 153, souligné par l'auteur).

"Commençons notre analyse par un examen de cette catégorie constitutive de tout récit, la catégorie des actions "(p. 155, souligné par l'auteur).

"La machine que nous sommes à lire progresse en opérant de multiples transformations de l'ordre visible ou dicible (ce qui d'ailleurs est le propre de toute littérature à fiction)" (p. 161).

Autant de "lois ", ou en tout cas de règles (n'entrons pas ici dans le délicat - mais pertinent - problème de la distinction entre lois et règles), assignables tantôt à toute écriture, tantôt à toute littérature, tantôt à tout récit, tantôt à toute littérature à fiction, et qui sont fort utiles à l'analyse des textes concrets. Je vois mal à vrai dire comment on pourrait procéder autrement. Mais sommes-nous ici très loin des analyses que proposent les formalistes? Propp sans doute cherche à dégager la structure générale du conte, mais c'est évidemment pour mieux décrire des contes particuliers $^{14}$; Greimas prétend scruter, à l'aide de sa sémantique structurale, l'univers de Bernanos ${ }^{15}$, et Todorov lui-même étudie Les Liaisons dangereuses ${ }^{16}$ ou le Décameron ${ }^{17}$.

Peut-être alors devrions-nous penser que le formalisme et le machinisme ne sont pas exclusifs l'un de l'autre, mais que celui-ci

14. Cf. Propp, V., Morphologie du Conte (traduit par M. Derrida), Paris, Éd du Seuil, 1965.

15. Cf. Greimas, A.J., Sémantique structurale, Paris, Larousse, 1966, p. 222.256.

16. Cf. Todorov, T., Littérature et signification, Paris, Larousse, 1967.

17. Cf. Todorov, T., La grammaire du Décaméron, La Haye, Mouton, 1969. 
présuppose et utilise celui-là. L'analyse machinique serait à la théorie de la littérature ce que la médecine est à la physiologie ou à la biologie : une science appliquée. Pas plus qu'à la présence ou à l'absence de méta-langage, la distinction ne tiendrait à la présence ou à l'absence de lois (ou de règles) générales, mais bien plutôt à une différence d'objectifs: les théoriciens formalistes rechercheraient les lois pour elles-mêmes, les machinistes les appliqueraient - celles-là ou d'autres - à l'étude de cas particuliers.

Comment d'ailleurs la description du fonctionnement d'une " machine " pourrait-elle faire l'économie d'une " mécanique " (au sens de : discipline qui étudie le mouvement et le fonctionnement des forces motrices et des machines) ? La théorie freudienne du travail onirique dont s'inspire le machiniste est bel et bien une théorie générale du rêve, et non pas la juxtaposition de descriptions particulières. De même le repérage et la mise en ordre des séries substitutives dans l'analyse du Quatuor requerrait à la limite quelque chose comme une théorie formelle des séries. Considérée de ce point de vue, l'opposition entre formalisme et machinisme tend à s'estomper, réduite au couple théorie/étude de cas.

\subsection{Lecture, jeu et description}

Mais peut-être n'avons-nous pas encore pris en considération l'élément le plus important. Peut-être la véritable originalité du machinisme ne se situe-t-elle pas, malgré l'insistance de Montpetit sur ce point, dans le rôle qu'il accorde au particulier, mais dans celui qu'il attribue à la lecture, occultée, semble-t-il, par le formalisme. Ce serait là bien sûr un tout autre critère de distinction, qu'on ne peut pas identifier purement et simplement à ceux dont il a déjà été question, mais certains passages du livre paraissent bien inviter à son adoption. L'étude d'Othello par exemple commence de la façon suivante :

"Un certain nombre de pages couvertes par un certain nombre de signes, avec entre eux des blancs et des espaces; tel se présente l'écrit à notre perception, le reste est lecture, c'est-à-dire traitement, travail, gestion" (p. 152).

Nous avons admis jusqu'ici que le machiniste visait à produire une analyse ou une description du texte. Mais est-ce bien là son objectif? Montpetit nous le présente au passage comme un " opérateur, apprêtant la machine textuelle, vérifiant ses rouages et 
mécanismes, et la faisant fonctionner " (p. 150-151). Peut-être ne s'agit-il pas tant de chercher à propos de l'œuvre littéraire un certain savoir, mais bien plutôt de la mettre en marche, de l'" actionner ", exactement comme on procède avec une automobile, un ouvre-boîte électrique ou une machine à boules. Le texte littéraire serait alors comparable à telle ouvre de Rauschenberg, dans laquelle une petite roulette est mise en mouvement par les bruits que produisent les spectateurs, l'intensité du bruit réglant la vitesse de la roulette. Le spectateur en pareil cas actionne littéralement l'œuvre, sans pour autant en chercher les lois générales de fonctionnement, sans la traiter comme cas particulier d'une loi physique :

"Le texte-machine, écrit Montpetit, est quelque chose dont on use $(.$.$) , la relation qui nous relie à lui n'est pas le dialogue,$ mais une opération active qui peut faire violence au texte en le traitant ». (p. 151).

Cette façon de voir est intimement - et d'ailleurs explicitement reliée à l'idée barthésienne du texte-pluriel :

"Si le texte-machine donne à lire (. . ), c'est davantage par sa malléabilité, par sa flexibilité et son aptitude à se prêter à divers usages toujours en fonctionnant» (p. 151).

On sait que, selon le Barthes de $S / Z$, qui entretient à l'endroit du projet d'une science de la littérarité les mêmes réserves que Montpetit (" le texte y perd sa différence "18), "l'enjeu du travail littéraire (...), c'est de faire du lecteur, non plus un consommateur, mais un producteur du texte ${ }^{19}$. Ce qui rejoint du même coup un véritable lieu commun de l'esthétique contemporaine, la mise en question de l'objet d'art comme pur spectacle, l'idée que le lecteur (ou le spectateur) est, pour reprendre l'expression de JeanLouis Galay, "co-effectueur du sens (en principe toujours pluriel) du texte $»^{20}$.

L'adoption d'une pareille optique produirait cependant un déplacement d'importance. D'abord on ne verrait plus très bien comment y situer l'idée d'une logique des séries substitutives. Mais

18. BARTheS, R., $S / Z$, Paris, Éd. du Seuil, 1970, p. 9.

19. Id.. p. 10.

20. GALAY, F.-L., "Esquisses pour une théorie figurale du discours ", in Poétique, no 20, 1974, p. 107. Cette connexion entre l'idée d'un lecteur actif et l'idée d'un texte-pluriel est une véritable constante de la méta-littérature contemporaine. 
surtout le machiniste, ainsi décrit, ne serait plus en quête de "rigueur " ou de "systématicité " - termes par lesquels pourtant Montpetit semblait dès le début de l'ouvrage (p. 5) caractériser l'objectif qu'il assignait à la critique littéraire -, mais il serait simplement un jouisseur, au même titre que celui qui prend plaisir à faire tourner la roulette de Rauschenberg. La démarche de $S / Z$, on le sait, conduit au "plaisir du texte".

Là est peut-être l'ambiguîté la plus sérieuse de l'entreprise de Montpetit. Car à y regarder de plus près, le machiniste n'est pas vraiment un "opérateur". Lui aussi écrit un texte, un autre, qui vise à démonter la machine littéraire, à en dégager les mécanismes, bref à en décrire les rouages. Il y a, qu'on le veuille ou pas, dans les analyses, d'ailleurs fort intéressantes, que Montpetit consacre à Durrell et à Shakespeare, une prétention à produire des énoncés vrais. Vérités relatives certes, mais n'est-ce pas le lot de toute vérité, y compris des vérités scientifiques? L'ambiguïté dont je parle peut être saisie sur le vif à l'apparente indifférence avec laquelle l'auteur propose tantôt de démonter la machine, tantôt de la faire fonctionner. Mais qui ne voit qu'on ne peut pas faire les deux à la fois ? Le moment où la critique s'écrit est différent du moment de la lecture jouisseuse.

Et si les énoncés du critique machiniste à propos d'un texte particulier prétendent être "vrais ", la véritable question est celle des procédures par lesquelles ils peuvent être validés ${ }^{21}$. Comment, par exemple, attester la présence d'une série substitutive? La notion - énigmatique - de " pluriel du texte " laisse quelquefois supposer que ces énoncés ne peuvent pas être validés : "On peut toujours, dit Montpetit, faire du texte un autre usage " (p. 151). Mais si toutes les lectures se valaient, il n'y aurait ni rigueur ni systématicité possibles. D'autres passages cependant inclinent à penser que le texte est construit de façon à produire certains effets et non pas certains autres : le lecteur, dit Montpetit ne peut qu'être "pris en charge " par la machination textuelle, "forcé d'y prendre place " (p. 150) ; et plus loin : "Un roman de tel genre se doit de produire nécessairement tels et tels effets » (p. 151). Or c'est apparemment cette contrainte textuelle, cette production nécessaire

21. Sur la question des procédures (possibles ou souhaitables) de validation des analyses de texte, voir les articles de Jean-Claude GARDIN, réunis in Les Analyses de discours, Neuchâtel, Delachaux et Niestlé, 1974. 
d'effets particuliers, que le machiniste voudrait décrire et dont il cherche à rendre compte :

"Ce que nous cherchons, ce sont les mécanismes qui permettent à cette écriture particulière de se dérouler et d'avoir cours, au texte de se poursuivre et de produire les effets pour lesquels il a été monté " (p. 158).

Bref, il y a dans le texte de Montpetit une tension entre deux entreprises incompatibles : celle, ludique, de jouer arbitrairement avec un texte, et celle, plus exigeante, de formuler des règles, d'énoncer des lois, de proposer une description du texte et des effets qu'il est susceptible de produire. Si certaines formules paraissent inviter à la première entreprise, on s'aperçoit bien qu'en réalité c'est la seconde qui est effectivement mise en œuvre à propos du Quatuor d'Alexandrie et d'Othello. Seul le refus, d'ailleurs purement verbal, du métalangage permet d'esquiver la question de la validation de cette description, élision qui à son tour engendre une série de confusions inextricables.

Et si le machiniste produit bien des analyses, alors le rôle particulier attribué au lecteur dans cette approche appelle quelque chose comme une théorie de la compétence du lecteur. Auquel cas la différence entre formalisme et machinisme tiendrait, contrairement à ce que dit Montpetit à plusieurs reprises, à ce que le premier - le formalisme - s'intéresse au texte même dans sa structure interne, alors que le second s'interroge sur ce qu'il advient du texte lorsqu'il est lu. Le machinisme rejoindrait par là une entreprise comme celle de Jean-Louis Galay :

"Notre description vise à imaginer un code d'enregistrement non pas de la surface discursive, mais de la lecture : il faut trouver le moyen de transcrire, au besoin grâce à un code approprié, ce que nous faisons quand nous lisons $"^{22}$.

Il n'y a pas de raison cependant pour qu'une telle "description" soit plus ludique ou moins formelle qu'une autre, ni en particulier pour qu'elle puisse faire l'économie d'un métalangage ${ }^{23}$.

22. GALAY, F.-L., art. cit., p. 408 (souligné par l'auteur). Voir aussi à ce propos l'article de Marianne CANTO-KLEIN et Nicole Ramognino (qui d'ailleurs suit d'assez près celui de Galay) : "Les faits sociaux sont pourvus de sens - Réflexions sur l'analyse de contenu ", in Connexions, no 11, 1974, p. 65.91.

23. Galay, par exemple, insiste beaucoup sur la nécessité de construire rigoureusement un tel métalangage. 


\subsection{Langue versus texte}

Ces remarques conduisent à ce qui, à mon sens, constitue le cœur du problème, la question du choix d'un modèle théorique. Si la distinction entre l'approche de Montpetit et celle d'un Todorov, par exemple, ne dépend ni du recours ou de l'absence de recours à un métalangage, ni vraiment d'une opposition entre l'universel et le particulier, ni de ceci que le machiniste jouerait avec le texte alors que le formaliste l'étudierait, mais d'une différence entre les éléments et les relations retenus par chacun comme pertinents pour l'analyse ou pour la description, alors cette distinction, idéalement, est celle de deux théories qui ne se donnent pas le même objet et qui n'utilisent pas les mêmes modèles formels.

Le machinisme serait alors, et l'on rejoint ici certaines des formules de Montpetit, l'étude des effets que produisent les structures textuelles sur les lecteurs (ou sur certaines catégories de lecteurs); il nécessiterait par conséquent une théorie des actes de lecture, une catégorisation des unités de lecture (ce que Barthes appelle les "lexies " $\left.{ }^{24}\right)$ et une mise au jour des codes utilisés par le lecteur (ce que Barthes essaie également de faire dans $S / Z$ ), de ceux par exemple qui lui permettent d' “ opérer " les mises en séries repérées par l'analyse machiniste.

Il est possible que le modèle théorique fourni par la linguistique structurale soit peu approprié à ce genre d'entreprise; non seulement parce que, ainsi qu'on le dit souvent, il privilégie l'unité - phrase et s'applique mal à des séquences plus longues, mais surtout parce qu'à première vue il permet difficilement de décrire quelque chose comme un acte ou quelque chose comme une série.

La véritable question que soulève le livre de Montpetit n'est pas, me semble-t-il, celle de l'opposition entre le formalisme et autre chose que le formalisme, mais celle de savoir quel modèle formel convient à l'analyse de la lecture littéraire et comment les énoncés conformes à ce modèle peuvent être rendus décidables. $\grave{A}$ moins de se contenter d'être un pur jeu et d'abandonner toute prétention à la rigueur et à l'adéquation, l'analyse machiniste doit idéalement du moins - se réclamer d'une théorie générale ; et ce serait de sa part pure faiblesse que de refuser a priori toute forma-

24. "Le signifiant-tuteur sera découpé en une série de courts fragments contigus, qu'on appellera ici des lexies, puisque ce sont des unités de lecture." (Barthes, R. $S / Z$, p. 20). 
lisation. Si le modèle structural est instatisfaisant, ce n'est certainement pas à cause de son caractère trop formel, mais peut-être seulement parce qu'il ne peut pas décrire le genre de relation que - plus ou moins confusément - on voudrait pouvoir analyser. En pareil cas, ou bien on change d'ambition, ou bien on change de modèle. Mais on peut difficilement prétendre mener l'analyse à bien sans aucun modèle. Ces remarques bien sûr ne résolvent pas le problème, mais peut-être contribuent-elles à le mieux poser. De toute façon, elles ne doivent évidemment pas être reçues comme une mise en question de l'intérêt du livre de Montpetit, mais comme la requête d'un prolongement à ce livre.

Université du Québec

à Trois-Rivières 